\title{
Glycaemic index of four commercially available breads in Malaysia.
}

\begin{abstract}
This study was carried out to determine the blood glucose response and glycaemic index (GI) values of four types of commercially available breads in Malaysia. Twelve healthy volunteers (six men, six women; body mass index, $21.9 \pm 1.6 \mathrm{~kg} / \mathrm{m} \mathrm{2}$; age, $22.9 \pm 1.7$ years) participated in this study. The breads tested were multi-grains bread (M-Grains), wholemeal bread (WM), wholemeal bread with oatmeal (WM-Oat) and white bread (WB). The subjects were studied on seven different occasions (four tests for the tested breads and three repeated tests of the reference food) after an overnight fast. Capillary blood samples were taken immediately before ( $0 \mathrm{~min})$ and 15, 30, 45, 60, 90 and $120 \mathrm{~min}$ after consumption of the test foods. The blood glucose response was obtained by calculating the incremental area under the curve. The GI values were determined according to the standardized methodology. Our results showed that the M-Grains and WM-Oat could be categorized as intermediate GI while the WM and WB breads were high GI foods, respectively. The GI of M-Grains (56 \pm 6.2$)$ and WM-Oat $(67 \pm 6.9)$ were significantly lower than the reference food (glucose; GI $=100)(\mathrm{P}<0.05)$. No significant difference in GI value was seen between the reference food and the GI of WM $(85 \pm 5.9)$ and WB $(82 \pm 6.5)(\mathrm{P}>0.05)$. Among the tested breads, the GI values of M-Grains and WM-Oat were significantly lower $(\mathrm{P}<0.05)$ than those of WM and WB. There was no relationship between the dietary fibre content of the bread with the incremental area under the curve $(r=0.15, \mathrm{P}=0.15)$ or their GI values $(\mathrm{r}=0.17, \mathrm{P}=0.12)$, indicating that the GI value of the test breads were unaffected by the fibre content of the breads. The result of this study will provide useful nutritional information for dieticians and the public alike who may prefer low-GI over high-GI foods.
\end{abstract}

Keyword: Blood glucose response; Bread; Dietary fibre; Glycaemic index. 